\title{
Practical approach to the sign problem at finite theta-vacuum angle
}

\section{Takahiro Sasaki*}

Department of Physics, Graduate School of Sciences, Kyushu University, Fukuoka 812-8581,

Japan

E-mail: sasaki@phys.kyushu-u.ac.jp

\section{Hiroaki Kouno}

Department of Physics, Saga University, Saga 840-8502, Japan

E-mail: kounohecc.saga-u.ac.jp

\section{Masanobu Yahiro}

Department of Physics, Graduate School of Sciences, Kyushu University, Fukuoka 812-8581, Japan

E-mail: yahiro@phys.kyushu-u.ac.jp

\begin{abstract}
We investigate a way of circumventing the sign problem in lattice QCD simulations with a thetavacuum term, using the Polyakov-loop extended Nambu-Jona-Lasinio (PNJL) model. We consider the reweighting method for the QCD Lagrangian after the $U_{\mathrm{A}}(1)$ transformation. In the Lagrangian, the $P$-odd mass term as a cause of the sign problem is minimized. In order to find a good reference system in the reweighting method, we estimate the average reweighting factor by using the two-flavor PNJL model and eventually find a good reference system.
\end{abstract}

31st International Symposium on Lattice Field Theory - LATTICE 2013

July 29 - August 3, 2013

Mainz, Germany

\footnotetext{
* Speaker.
} 


\section{Introduction}

The existence of instanton solution requires QCD Lagrangian with the theta vacuum:

$$
\mathscr{L}=\sum_{f} \bar{q}_{f}\left(\gamma_{v} D_{v}+m_{f}\right) q_{f}+\frac{1}{4 g^{2}} F_{\mu \nu}^{a} F_{\mu \nu}^{a}-i \theta \frac{1}{64 \pi^{2}} \varepsilon_{\mu \nu \sigma \rho} F_{\mu \nu}^{a} F_{\sigma \rho}^{a},
$$

in Euclidean spacetime. Hereafter, we will consider two-flavor QCD and assume isospin symmetry, $m_{u}=m_{d}=m_{0}$. Though the angle $\theta$ can take any arbitrary value theoretically, experimental measurements of neutron dipole moment give the upper limit, $|\theta|<10^{-9}[1]$. Why should $\theta$ be so small? This long-standing puzzle is called the strong $C P$ problem.

Since the upper limit is determined only at zero temperature, the behavior is nontrivial for finite temperature. Hence the first-principle lattice simulation is needed, but it has the sign problem for finite $\theta$. After making $U_{A}(1)$ transformation,

$$
q=e^{i \gamma_{5} \frac{\theta}{4}} q^{\prime}
$$

$\theta$ dependence appears only through the mass term,

$$
m_{0}(\theta)=m_{0} \cos (\theta / 2)+m_{0} i \gamma_{5} \sin (\theta / 2),
$$

in the transformed Lagrangian,

$$
\mathscr{L}=\sum_{f} \bar{q}_{f}\left(\gamma_{v} D_{v}+m_{0}(\theta)\right) q_{f}+\frac{1}{4 g^{2}} F_{\mu \nu}^{a} F_{\mu \nu}^{a}
$$

The $P$-odd mass term including $i \gamma_{5}$ makes the fermion determinant complex.

Because of the sign problem, we should perform a reweighting method in lattice simulations. The vacuum expectation value of operator $\mathscr{O}$ is obtained by

$$
\begin{aligned}
\langle\mathscr{O}\rangle & =\int \mathscr{D} A \mathscr{O} \operatorname{det} \mathscr{M}(\theta) e^{-S_{g}} \\
& =\int \mathscr{D} A \mathscr{O}^{\prime} \operatorname{det} \mathscr{M}_{\mathrm{ref}}(\theta) e^{-S_{g}}
\end{aligned}
$$

with the gluon part $S_{g}$ of the QCD action and

$$
\mathscr{O}^{\prime} \equiv R(\theta) \mathscr{O}, \quad R(\theta) \equiv \frac{\operatorname{det} \mathscr{M}(\theta)}{\operatorname{det} \mathscr{M}_{\mathrm{ref}}(\theta)},
$$

where $R(\theta)$ is the reweighting factor and $\operatorname{det} \mathscr{M}_{\mathrm{ref}}(\theta)$ is the fermion determinant of the reference theory that has no sign problem. The simplest candidate of the reference theory is the theory in which the $\theta$-odd term is neglected in the mass term 1.3. We refer to this reference theory as reference A in this paper. As discussed in Ref. [2], reference A may be a good reference theory for small and intermediate $\theta$, but not for large $\theta$ near $\pi$. In reference $\mathrm{A}$, the limit of $\theta=\pi$ corresponds to the chiral limit for $\operatorname{det} \mathscr{M}_{\text {ref }}$ that is hard for LQCD simulations to reach.

The expectation value of $R(\theta)$ in the reference theory is obtained by

$$
\langle R(\theta)\rangle=\frac{Z}{Z_{\text {ref }}}
$$


where $Z\left(Z_{\text {ref }}\right)$ is the partition function of the original (reference) theory. The average reweighting factor $\langle R(\theta)\rangle$ is a good index for the reference theory to be good; the reference theory is good when $\langle R(\theta)\rangle=1$.

In this work, we estimate $\langle R(\theta)\rangle$ with the two-flavor PNJL model in order to find a good reference theory. We find that reference A is good only for small $\theta$, so propose a good reference theory that satisfies $\langle R(\theta)\rangle \approx 1$. This work is based on the Ref. [3].

\section{Model setting}

The two-flavor PNJL Lagrangian with the $\theta$-dependent anomaly term is obtained in Euclidean spacetime by

$$
\begin{aligned}
\mathscr{L}= & \bar{q}\left(\gamma_{v} D_{v}+m_{0}\right) q-G_{1} \sum_{a=0}^{3}\left[\left(\bar{q} \tau_{a} q\right)^{2}+\left(\bar{q} i \gamma_{5} \tau_{a} q\right)^{2}\right] \\
& -8 G_{2}\left[e^{i \theta} \operatorname{det} \bar{q}_{\mathrm{R}} q_{\mathrm{L}}+e^{-i \theta} \operatorname{det} \bar{q}_{\mathrm{L}} q_{\mathrm{R}}\right]+\mathscr{U}\left(T, \Phi, \Phi^{*}\right),
\end{aligned}
$$

where $D_{v}=\partial_{v}-i \delta_{v 4} A_{4}^{a} / \lambda_{a} / 2$ with the Gell-Mann matrices $\lambda_{a}$. The current quark mass $m_{0}$ satisfies $m_{0}=m_{u}=m_{d}$, and $\tau_{0}$ and $\tau_{a}(a=1,2,3)$ are the $2 \times 2$ unit and Pauli matrices in the flavor space, respectively. The parameter $G_{1}$ denotes the coupling constant of the scalar and pseudoscalar-type four-quark interactions, while $G_{2}$ stands for that of the Kobayashi-Maskawa-'t Hooft determinant interaction [4] 5] where the matrix indices run in the flavor space. The Polyakov-loop $\Phi$ and its conjugate $\Phi^{*}$ are determined in the Euclidean space by $\Phi=\frac{1}{3} \operatorname{tr}_{\mathrm{c}}(L), \Phi^{*}=\frac{1}{3} \operatorname{tr}_{\mathrm{c}}(\bar{L})$, where $L=\exp \left(i A_{4} / T\right)$ with $A_{4} / T=\operatorname{diag}\left(\phi_{r}, \phi_{g}, \phi_{b}\right)$ in the Polyakov gauge. We use the Polyakov potential $\mathscr{U}$ of Ref. [6]:

$$
\begin{aligned}
& \mathscr{U}=T^{4}\left[-\frac{a(T)}{2} \Phi^{*} \Phi+b(T) \ln \left(1-6 \Phi \Phi^{*}+4\left(\Phi^{3}+\Phi^{* 3}\right)-3\left(\Phi \Phi^{*}\right)^{2}\right)\right], \\
& a(T)=a_{0}+a_{1}\left(\frac{T_{0}}{T}\right)+a_{2}\left(\frac{T_{0}}{T}\right)^{2}, b(T)=b_{3}\left(\frac{T_{0}}{T}\right)^{3} .
\end{aligned}
$$

Under the $U_{A}(1)$ transformation (1.2), the Lagrangian density is then rewritten with $q^{\prime}$ as

$$
\mathscr{L}=\bar{q}^{\prime}\left(\gamma_{v} D_{v}+m_{0}(\theta)\right) q^{\prime}-G_{+}\left[\left(\bar{q}^{\prime} q^{\prime}\right)^{2}+\left(\bar{q}^{\prime} i \gamma_{5} \vec{\tau} q^{\prime}\right)^{2}\right]-G_{-}\left[\left(\bar{q}^{\prime} \vec{\tau} q^{\prime}\right)^{2}+\left(\bar{q}^{\prime} i \gamma_{5} q^{\prime}\right)^{2}\right]+\mathscr{U},
$$

where $G_{ \pm}=G_{1} \pm G_{2}$.

Applying the saddle-point approximation to the path integral in the partition function, one can get the average reweighting factor $\langle R(\theta)\rangle$,

$$
\begin{gathered}
\langle R(\theta)\rangle \approx R_{A} R_{B} \\
R_{A}=\sqrt{\frac{\operatorname{det} H_{\text {ref }}}{\operatorname{det} H}}, R_{B}=e^{-\beta V\left(\Omega-\Omega_{\mathrm{ref}}\right)},
\end{gathered}
$$

where $\beta=1 / T$ and $\Omega\left(\Omega_{\text {ref }}\right)$ is the thermodynamic potential at the mean-field level in the original (reference) theory [3]. $H\left(H_{\text {ref }}\right)$ is the Hessian matrix in the original (reference) theory defined by [7] 8

$$
H_{i j}=\frac{\partial^{2} \Omega}{\partial \phi_{i}^{\prime} \partial \phi_{j}^{\prime}}, \quad\left\{\phi_{i}^{\prime}\right\}=\left\{\sigma^{\prime}, \eta^{\prime}, \vec{a}^{\prime}, \vec{\pi}^{\prime}\right\}
$$


with the quark-condensates

$$
\sigma^{\prime}=\left\langle\bar{q}^{\prime} q^{\prime}\right\rangle, \quad \eta^{\prime}=\left\langle\bar{q}^{\prime} i \gamma_{5} q^{\prime}\right\rangle, \vec{a}^{\prime}=\left\langle\bar{q}^{\prime} \vec{\tau} q^{\prime}\right\rangle, \quad \vec{\pi}^{\prime}=\left\langle\bar{q}^{\prime} i \gamma_{5} \vec{\tau} q^{\prime}\right\rangle
$$

The four-dimensional volume $\beta V$ is obtained by $\beta V=\left(N_{x} / N_{\tau}\right)^{3} T^{-4}$ for the $N_{x}^{3} \times N_{\tau}$ lattice. Here we consider $N_{x} / N_{\tau}=4$ as a typical example, following Refs. [7 8$]$.

We consider the following reference theory that has no sign problem:

$$
\mathscr{L}_{\text {ref }}=\bar{q}^{\prime}\left(\gamma_{v} D_{v}+m_{\mathrm{ref}}(\theta)\right) q^{\prime}-G_{+}\left[\left(\bar{q}^{\prime} q^{\prime}\right)^{2}+\left(\bar{q}^{\prime} i \gamma_{5} \vec{\tau} q^{\prime}\right)^{2}\right]-G_{-}\left[\left(\bar{q}^{\prime} \vec{\tau} q^{\prime}\right)^{2}+\left(\bar{q}^{\prime} i \gamma_{5} q^{\prime}\right)^{2}\right]+\mathscr{U} .
$$

Here $m_{\text {ref }}(\boldsymbol{\theta})$ is $\theta$-even mass defined below. We consider three examples as $m_{\text {ref }}(\theta)$.

\section{Numerical results}

If some reference system satisfies the condition $\langle R(\theta)\rangle \approx 1$, one can say that the reference system is good. As a typical example of the condition, we consider the case of $0.5 \lesssim\langle R(\theta)\rangle \lesssim 2$. This condition seems to be the minimum requirement. The discussion made below is not changed qualitatively, even if one takes a stronger condition.

The first example is reference A defined by

$$
\begin{aligned}
m_{\mathrm{ref}}(\boldsymbol{\theta}) & \equiv m_{\mathrm{A}}(\boldsymbol{\theta}) \\
& =m_{0} \cos (\boldsymbol{\theta} / 2) .
\end{aligned}
$$

In this case, the $P$-odd mass is simply neglected from the original Lagrangian (2.4).

Figure1(a) shows $\theta$ dependence of $\langle R(\theta)\rangle$ at $T=100 \mathrm{MeV}$. The solid line stands for $\langle R(\theta)\rangle$, while the dashed (dotted) line corresponds to $R_{\mathrm{A}}\left(R_{\mathrm{B}}\right)$. This temperature is lower than the chiral transition temperature in the original theory that is $212 \mathrm{MeV}$ at $\theta=0$ and $204 \mathrm{MeV}$ at $\theta=\pi$. As $\theta$ increases from zero, $\langle R(\theta)\rangle$ also increases and exceeds 2 at $\theta \approx 1$.2. Reference $\mathrm{A}$ is thus good for $\theta \lesssim 1.2$.

Figure 1(b) shows $\theta$ dependence of pion mass $\tilde{M}_{\pi}$ at $T=100 \mathrm{MeV}$. Since $P$ symmetry is broken at finite $\theta, P$-even modes and $P$-odd modes are mixed with each other for each meson. Hence, $\tilde{M}_{\pi}$ is defined by the lowest pole mass of the inverse propagator in the isovector channel[3]. The solid (dashed) line denotes $\tilde{M}_{\pi}$ in the original (reference A) system. At $\theta=\pi, \tilde{M}_{\pi}$ is finite in the original system, but zero in reference $\mathrm{A}$. As a consequence of this property, $R_{\mathrm{A}}$ and $\langle R(\theta)\rangle$ vanish at $\theta=\pi$; see Fig. 1 (1a). This indicates that reference A breaks down at $\theta=\pi$.

The second example is reference $\mathrm{B}$ defined by

$$
\begin{aligned}
m_{\mathrm{ref}}(\theta) & \equiv m_{\mathrm{B}}(\theta) \\
& =m_{0} \cos (\theta / 2)+\frac{1}{\alpha}\left\{m_{0} \sin (\theta / 2)\right\}^{2} .
\end{aligned}
$$

In this case, we have added the $m_{0}^{2}$-order correction due to the $P$-odd quark mass. Here $\alpha$ is a parameter with mass dimension, so we simply choose $\alpha=M_{\pi}$. The coefficient of the correction term is $m_{0}^{2} / M_{\pi}=0.129 \mathrm{MeV}$.

The same analysis is made for reference B in Fig. $2 \tilde{M}_{\pi}$ in reference B well reproduces that in the original theory for any $\theta$ and $\langle R\rangle$ satisfies the condition $0.5 \lesssim R \lesssim 2$ for all $\theta$. Since $R_{H} \sim 1$ 

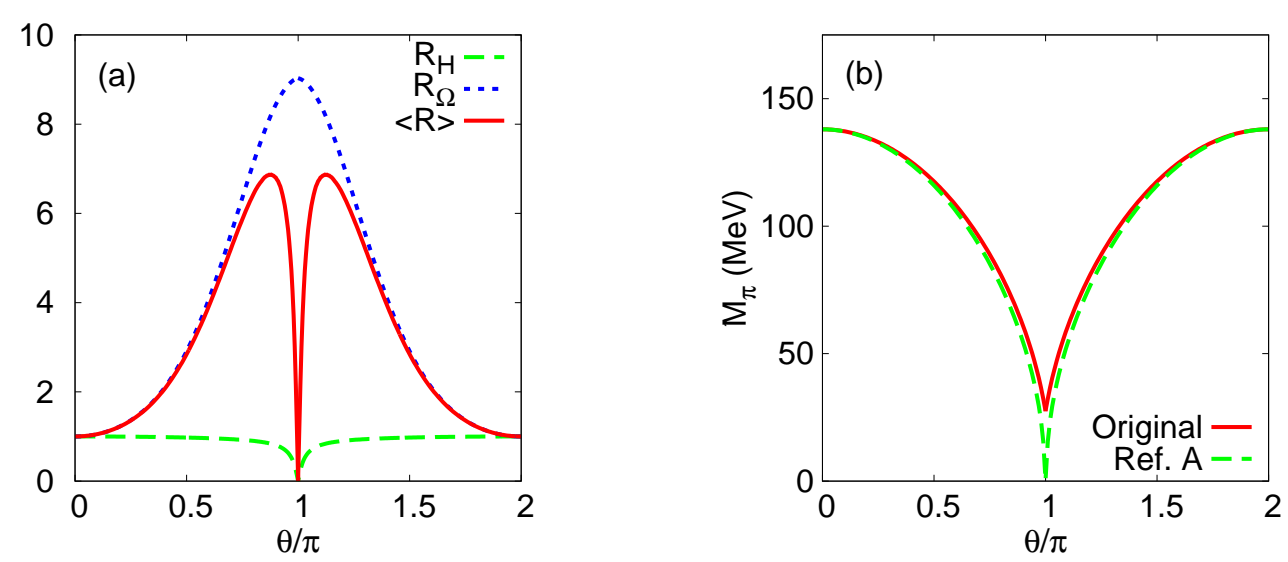

Figure 1: $\theta$ dependence of (a) the average reweighting factor and (b) $\tilde{M}_{\pi}$ at $T=100 \mathrm{MeV}$ for the case of reference A.
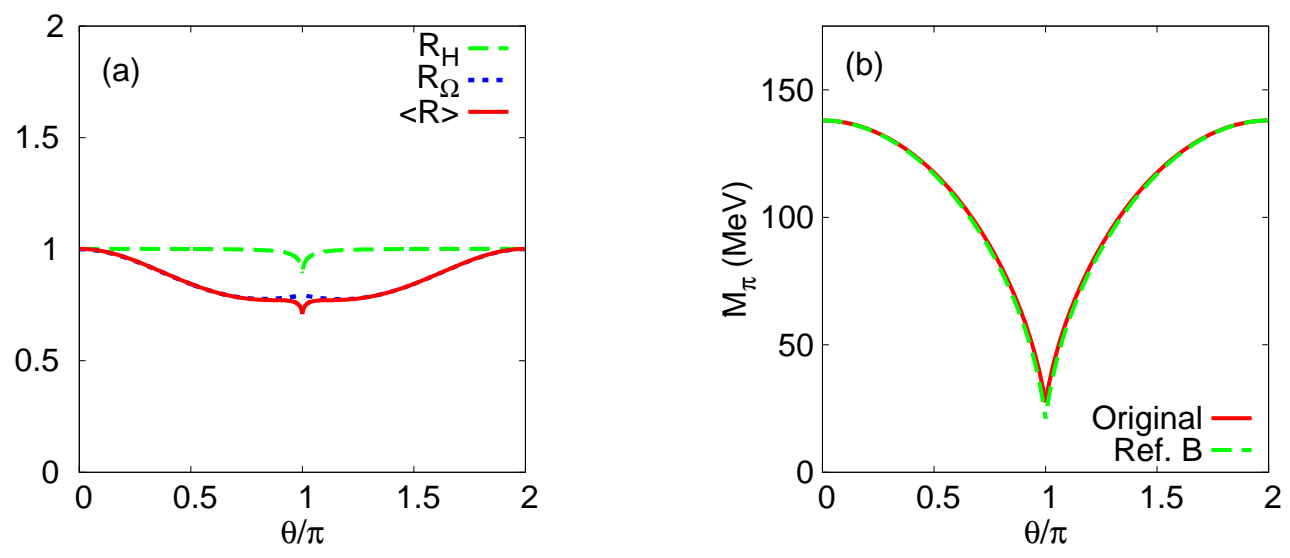

Figure 2: $\theta$ dependence of (a) the average reweighting factor and (b) $\tilde{M}_{\pi}$ at $T=100 \mathrm{MeV}$ for the case of reference $B$.

in the most region of $\theta,\langle R(\theta)\rangle$ is governed by $R_{\Omega}$. Around $\theta=\pi, R_{H}$ becomes small but still has a nonzero value because $\tilde{M}_{\pi} \neq 0$ even at $\theta=\pi$ in reference B. Therefore, the simple estimation for $m_{\text {ref }}(\theta)$ (3.2) gives an available reference.

Finally we consider reference $\mathrm{C}$. The pion mass $\tilde{M}_{\pi}(\theta)$ at finite $\theta$ is estimated from the chiral Lagrangian and $1 / N_{c}$ analysis [9]:

$$
\tilde{M}_{\pi}^{2}(\theta)=\frac{\left|\sigma_{0}\right|}{f_{\pi}^{2}}\left[m_{0}|\cos (\theta / 2)|+\frac{m_{0} M_{\pi}^{2}}{M_{\eta^{\prime}}^{2}} \sin ^{2}(\theta / 2)\right] .
$$

where $\sigma_{0}$ is the chiral condensate at $T=\theta=0$. Interpreting a $\theta$ dependent mass from this result, reference $\mathrm{C}$ is defined by

$$
\begin{aligned}
m_{\mathrm{ref}}(\theta) & \equiv m_{\mathrm{C}}(\theta) \\
& =m_{0} \cos (\theta / 2)+\frac{m_{0} M_{\pi}^{2}}{M_{\eta^{\prime}}^{2}} \sin ^{2}(\theta / 2)
\end{aligned}
$$



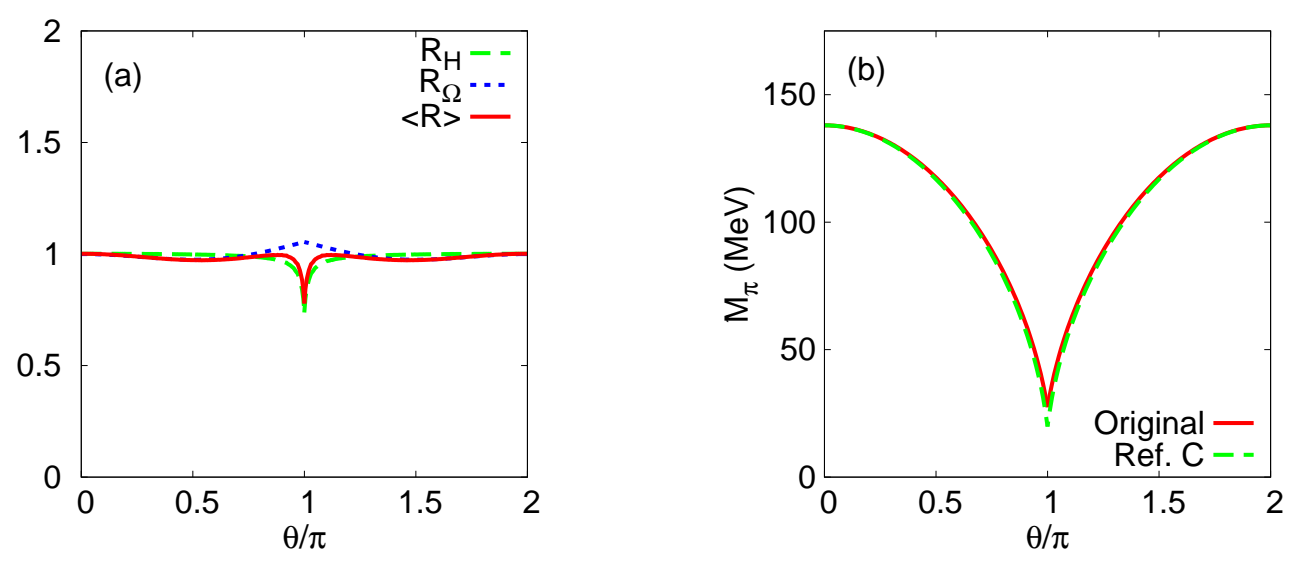

Figure 3: $\theta$ dependence of (a) the average reweighting factor and (b) $\tilde{M}_{\pi}$ at $T=100 \mathrm{MeV}$ for the case of reference $\mathrm{C}$.

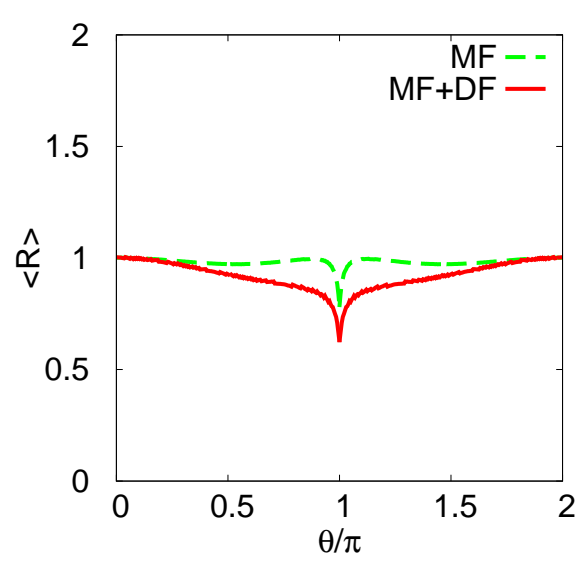

Figure 4: $\theta$ dependence of the average reweighting factor at $T=100 \mathrm{MeV}$ for the case of reference C. Solid and dashed lines correspond to the result with and without dynamical pion fluctuation, respectively.

This case also has the $m_{0}^{2}$-order correction, but $\alpha$ is different from reference $\mathrm{B}$. The coefficient of the correction term is $m_{0} M_{\pi}^{2} / M_{\eta^{\prime}}^{2}=0.114 \mathrm{MeV}$.

As shown in Fig. B(b), $\tilde{M}_{\pi}$ in reference $\mathrm{C}$ slightly underestimates that of the original theory at small and intermediate $\theta$. However, in Fig. 3 a), $\langle R(\theta)\rangle$ satisfies the condition $0.5 \lesssim\langle R(\theta)\rangle \lesssim 2$ for all $\theta$. Therefore we can think that reference $\mathrm{C}$ is a good reference system for any $\theta$.

Beyond the mean-field approximation, we estimate an effect of dynamical pion fluctuations by modifying the thermodynamic potential to

$$
\Omega=\Omega_{\mathrm{MF}}+\Omega_{\mathrm{DF}},
$$

where $\Omega_{\mathrm{MF}}$ is the thermodynamic potential with the mean-field level. $\Omega_{\mathrm{DF}}$ is the potential due to dynamical pion fluctuations [8],

$$
\Omega_{\mathrm{DF}}=3 \int \frac{d^{3} p}{(2 \pi)^{3}} T \ln \left(1-e^{-\beta E_{\pi}}\right)
$$

where $E_{\pi}=\sqrt{\vec{p}^{2}+\tilde{M}_{\pi}^{2}}$. 
Figure 4 shows $\theta$ dependence of $\langle R(\theta)\rangle$ at $T=100 \mathrm{MeV}$ for the case of reference $\mathrm{C}$. The solid and dashed lines correspond to results with and without dynamical pion fluctuations, respectively. The effect makes $\langle R\rangle$ a little smaller, and hence the reference $\mathrm{C}$ becomes slightly worse. However, the modification is small, indicating that $\langle R\rangle$ is well evaluated by the mean-field approximation

\section{Summary and discussion}

We have investigated a way of circumventing the sign problem in LQCD simulations with finite $\theta$, using the PNJL model. We have considered the reweighting method for the transformed Lagrangian (1.4). In the Lagrangian, the sign problem is minimized, since the $P$-odd mass is much smaller than $\Lambda_{\mathrm{QCD}}$. Another key is which kind of reference system satisfies the condition $\langle R(\theta)\rangle \approx 1$. We have then estimated $\langle R(\theta)\rangle$ by using the two-flavor PNJL model and have found that reference $\mathrm{C}$ may be a good reference system in the reweighting method.

Since the present proposal is based on the model analysis, it is then not obvious whether the proposal really works in lattice simulations. Therefore, the proposal should be directly tested by lattice simulations. A similar test was made for two-flavor QCD with finite quark chemical potential $\mu[10,8 \rrbracket$ where lattice simulations have the sign problem. The average reweighting factor, i.e., the average phase factor, was evaluated by lattice simulations at $\mu / T<1$ for $T$ around the critical temperature of the deconfinement transition [10]. The PNJL model well reproduces the lattice result, when the dynamical correction due to mesonic fluctuations is made to the mean-field model calculation [8]. It is thus interesting that the present proposal is directly tested by lattice simulations.

\section{Acknowledgments}

This work was supported by JSPS KAKENHI Grant No. 23-2790.

\section{References}

[1] C. A. Baker, et al., Phys. Rev. Lett. 97, 131801 (2006).

[2] T. Sasaki, J. Takahashi, Y. Sakai, H. Kouno, and M. Yahiro, Phys. Rev. D 85, 056009 (2012).

[3] T. Sasaki, H. Kouno, and M. Yahiro. Phys. Rev. D 87056003 (2013).

[4] M. Kobayashi, and T. Maskawa, Prog. Theor. Phys. 44, 1422 (1970); M. Kobayashi, H. Kondo, and T. Maskawa, Prog. Theor. Phys. 45, 1955 (1971).

[5] G. 't Hooft, Phys. Rev. Lett. 37, 8 (1976); Phys. Rev. D 14, 3432 (1976); 18, 2199(E) (1978).

[6] S. Rößner, C. Ratti, and W. Weise, Phys. Rev. D 75, 034007 (2007).

[7] J. O. Andersen, L. T. Kyllingstad, and K. Splittroff, J. High Energy Phys. 01, 055 (2010).

[8] Y. Sakai, T. Sasaki, H. Kouno, and M. Yahiro, Phys. Rev. D 82, 096007 (2010).

[9] M. A.Metlitski, and A. R. Zhitnitsky, Nucl. Phys. B731, 309 (2005); Phys. Lett. B 633, 721 (2006).

[10] M. D’Elia and F. Sanfilippo, Phys. Rev. D 80, 014502 (2009). 